\title{
Liebe Kolleginnen, Liebe Kollegen!
}

Die Österreichische Gesellschaft für Sportmedizin und Prävention (ÖGSMP) ist die Fachgesellschaft der Österreichischen Sportärzte. Sie ist verantwortlich für die Inhalte der Ausbildungsrichtlinien für das Sportarztdiplom der Österreichischen Ärztekammer. Die meisten Sportmedizinischen Institute beziehungsweise Untersuchungsstellen, die die Betreuung sowohl von PatientInnen wie auch SportlerInnen übernehmen, werden von Mitgliedern des Sportärzteverbandes geleitet. Auch die Betreuung im niedergelassenen Bereich erfolgt größtenteils durch Kolleglnnen, die Mitglieder unseres Verbandes sind.

Die vielen Spezialisten, die im Vorstand der ÖGSMP vertreten sind und die die einzelnen Fachgesellschaften repräsentieren, dokumentieren die Breite unseres Verbandes. Wir können gemeinsam mit den Experten des klinischen wie auch des extramuralen Bereichs Vorschläge beziehungsweise Konzepte anbieten, die es erlauben mitzuhelfen, die derzeitigen Probleme des Bewegungsmangels in den Griff zu bekommen.

Denn die Lifestyle-Veränderungen in unserer Gesellschaft stellen sowohl gesundheits- wie auch wirtschaftspolitisch große Anforderungen an die Verantwortlichen. Die gesundheitlichen Veränderungen durch den Bewegungsmangel, die schon im Kindesalter auffällig werden, erfordern strategische Maßnahmen im Sinne von therapeutischen Konzepten. Aus diesem Grund sehe ich die Sportmedizin als „Trendmedizin“ unserer Zeit. Wenn sich auch viele Berufsgruppen in den Sektor „Gesundheit“ drängen, ist es doch die Domäne und Verantwortung der Sportmedizin, das richtige Rezept für Belastung und Beanspruchung zu verordnen.

Die Verordnung von Bewegungstherapie auf Rezept wäre zudem eine kostengünstige und effektivere Maßnahme, um Gefäßerkrankungen, Übergewicht, Typ II Diabetes und Probleme am Bewegungsapparat, aber auch manche psychosomatische Erkrankungen in den Griff zu bekommen. Eine Maßnahme, die sich die Politik sehr gut überlegen sollte, da sie sowohl präventiv wie auch therapeutisch wirken würde und zur Senkung der Medikamenten- und Behandlungskosten beitragen würde.

Die ÖGSMP hat mit der Installierung einer Arbeitsgruppe für Medizinische Trainingslehre und Therapie die ersten Schritte und damit die Voraussetzung für die Umsetzung dieser Idee gesetzt.

Auch das Thema Doping im Sport macht nicht an Österreichs Grenzen halt. Durch das neue Anti-Doping-Gesetz versucht die Regierung, der Anwendung unerlaubter Maßnahmen im Sport zu begegnen. Zur Unterstützung hat die ÖGSMP eine Arbeitsgruppe installiert, die sich mit dieser Thematik, vor allem aber auch mit Anti-Doping-Maßnahmen beschäftigt. Sowohl in der Ausbildung, als auch für die diplomierten SportärztInnen wird es Informationen über die Handhabung des Gesetzes, über die Gefahren des Dopings und über alternative, legale Leistung steigernde Maßnahmen geben.

Überhaupt bemüht sich unsere Gesellschaft, die Ausbildung zum Sportarzt/Ärztin laufend zu verbessern und die Inhalte an neue Erkenntnisse anzupassen.

Ein wichtiges Anliegen unserer Gesellschaft ist es auch, Leitlinien für Untersuchungsalgorithmen zu schaffen, um so eine möglichst einheitliche und transparente Betreuung von PatientInnen und Sportlerlnnen zu gewährleisten. Verbesserungswürdig scheinen mir auch die Akzeptanz und die Präsenz der Österreichischen Sportmedizin in der Öffentlichkeit, in den Medien und in der Politik. Es ist also Corporate Idendity und PR nach Innen und Außen gefragt.

Diese Verbesserung können wir allerdings nur gemeinsam, mit ihrer, der Unterstützung aller SportärztInnen schaffen.

Im Spitzensport zeigt sich, dass die Verbände, die ein interdisziplinäres Konzept und eine Kooperation mit der österreichischen Sportmedizin haben, diese auch im internationalen Vergleich erfolgreich sind. Es muss daher unser Ziel sein, eine Zusammenarbeit mit der BSO und den Verbänden zu suchen und unsere Unterstützung anzubieten beziehungsweise zu intensivieren.

Unsere Bemühungen müssen und werden sich aber auch weiterhin auf die sportliche Zukunft, den Nachwuchssport konzentrieren, da jugendliche Leistungssportler, die die sportmedizinische Betreuung gewohnt sind, auch später auf sie nicht verzichten werden. Für all dies soll das neue Journal der Österreichischen Sportmedizin eine Informationsplattform in Form von wissenschaftlichen, praxisrelevanten und standespolitischen Beiträgen sein, das dazu beitragen kann, sportmedizinische Themen einer breiten Interessentengruppe zugänglich zu machen. Die ÖGSMP wird sich auch weiterhin bemühen, das Ansehen der österreichischen Sportmedizin hochzuhalten beziehungsweise zu verbessern.

\section{Univ.-Prof. Dr. Peter Schober}

